\title{
Lymph Node Dissection During the Surgical Treatment of Renal Cancer in the Modern Era
}

\author{
Guilherme Godoy, Rebecca L. O’Malley, Samir S. Taneja \\ Urologic Oncology Program, Department of Urology, New York University School of Medicine, \\ New York, NY, USA
}

\begin{abstract}
The increasing use of routine CT scan, along with advances in imaging technology, have facilitated the early diagnosis of incidental renal masses. This has resulted in the reduction in the rate of metastatic disease diagnosis.

Although surgery remains the mainstay in the treatment of renal tumors, the decreasing incidence of lymph node involvement has created controversy regarding the importance and the ideal extent of lymph node dissection, formerly considered mandatory at the time of radical nephrectomy. In this review, we critically assessed the role of lymph node dissection at the time of radical nephrectomy. To date, randomized trials have failed to show a benefit of lymph node dissection when broadly employed. This is likely due to the low prevalence of lymph node metastasis at the time of presentation, the unpredictable pattern of lymph node metastasis from renal tumors, and the continued downward stage migration of the disease. As a result, lymph node dissection for renal cancer is currently not recommended in the absence of gross lymphadenopathy. In high risk patients, lymph node dissection may be considered, but it remains controversial and more clinical evidence is warranted. Extended lymph node dissection is still recommended in individuals with isolated gross nodal disease or those with lymphadenopathy at the time of cytoreductive surgery prior to systemic therapy. A practical approach is summarized in an algorithm form.
\end{abstract}

Key words: kidney neoplasms; nephrectomy; lymph nodes; lymph node excision; disease management; review Int Braz J Urol. 2008; 34: 132-42

\section{INTRODUCTION}

The role of lymphadenectomy in the surgical management of renal cell carcinoma (RCC) still remains controversial among urologists. In an age of continuous decreasing incidence of lymph node metastasis, the broad application of lymph node dissection (LND) has recently been criticized by several authors due to the absence of demonstrated therapeutic benefit, as reported in the European Organization for Research and Treatment of Cancer trial number 30881 (EORTC 30881), the only prospective trial that compared the outcomes of radical nephrectomy alone versus associated with LND (1). In order to define the optimal contemporary approach to LND during the surgical management of RCC, we analyzed the most relevant data published regarding this issue. The review of retrospective studies can be challenging due to inherent bias such as variable inclusion criteria, disparate study designs, variable LND technique, use of inconsistent staging systems and selection bias. For this analysis, three important factors should be considered: 1) the prevalence of lymph node metastasis; 2) the morbidity associated with LND; and 3) 
the benefit of performing LND in these cases. Once a balance between all these parameters is reached, the role of LND in clinical practice probably becomes more evident. In this review, we evaluate the prevalence of lymph node metastasis, the efficacy of LND, and present a rational algorithm for the selection of suitable candidates for LND at the time of radical nephrectomy.

\section{PREVALENCE, RISK FACTORS AND RELEVANCE}

The reported overall incidence of lymph node metastasis in renal cancer in surgical and autopsy series is approximately $20 \%$, but there is a significant variability reported in the literature (2). As seen in Table-1, the incidence of identified lymph node

Table 1 - Overall Prevalence of $\mathrm{N}+$.

\begin{tabular}{|c|c|c|c|c|c|}
\hline Year & Author (Ref.) & Study Design & $\begin{array}{l}\text { Number of Cases / } \\
\text { Groups Included }\end{array}$ & $\begin{array}{c}\text { LND } \\
\text { Technique }\end{array}$ & Prevalence of $\mathbf{N}+$ \\
\hline 1982 & Saitoh (15) & autopsy & $1828 /$ all stages & autopsy & $63.6 \%$ \\
\hline 1997 & Johnsen \& Hellsten (9) & autopsy & 554/ all stages & autopsy & $14.0 \%$ \\
\hline 1969 & Robson (14) & retrospective & 88/ all stages & extended & $22.7 \%$ \\
\hline 1971 & Skinner (18) & retrospective & 309/ all stages & regional & $19.4 \%$ \\
\hline 1978 & De Kernion (4) & retrospective & 86/ Tany N+ or M+ & regional & $22.0 \%$ \\
\hline 1979 & Waters (23) & retrospective & $130 /$ all stages & regional & $24.0 \%$ \\
\hline 1980 & Peters (13) & retrospective & 356/ all stages & extended & $10.3 \%$ \\
\hline 1981 & Sigel (24) & retrospective & & extended & $38.0 \%$ \\
\hline 1982 & Siminovitch (17) & retrospective & 102/ T1-1N0M0 & mixed & $8.8 \%$ \\
\hline 1986 & Golimbu (7) & retrospective & $326 /$ all stages & extended & $7.9 \%$ \\
\hline 1990 & Giuliani (6) & retrospective & 200/ all stages & extended & $24.0 \%$ \\
\hline 1990 & Tsukamoto (21) & retrospective & $102 /$ all stages & extended & $21.0 \%$ \\
\hline 1990 & Studer (19) & retrospective & $163 /$ all stages & extended & $14.1 \%$ \\
\hline 1991 & Herrlinger (8) & retrospective & $511 /$ all stages & extended & $17.5 \%$ \\
\hline 1992 & Ditonno (5) & retrospective & 97/ all stages & extended & $6.2 \%$ \\
\hline 1997 & Giberti (25) & retrospective & 328/ all stages & regional/extended & $20.4 \%$ \\
\hline 1999 & Schafhauser (16) & retrospective & 1035/ TanyN0M0 & mixed & $14.1 \%$ \\
\hline 1999 & Moudoni (11) & retrospective & 68/ TanyNanyM0 & -- & $20.0 \%$ \\
\hline 2001 & Minervini (10) & retrospective & 167/ TanyN0M0 & regional & $5.0 \%$ \\
\hline 2001 & Vasselli (22) & retrospective & 154/ TanyNanyM+ & mixed & $46.0 \%$ \\
\hline 2003 & Terrone (20) & retrospective & $725 /$ all stages & extended & $13.6 \%(>12 n=20.4 \%)$ \\
\hline 2003 & Pantuck (12) & retrospective & 900/ all stages & mixed & $14.3 \%$ \\
\hline 2004 & Blute (3) & retrospective & 2028/ all stages (pM0) & extended & $4.4 \%$ \\
\hline 1999 & Blom-EORTC (1) & prospective & 731/ T1-3N0M0 & extended & $3.3 \%$ \\
\hline
\end{tabular}

$L N D=$ lymph node dissection $; n=$ nodes $;$ EORTC = European Organization for Research and Treatment of Cancer. 
metastasis ranges from 3\% in surgical series, up to $63.6 \%$ in autopsy series $(1,3-25)$. The wide variation may be explained by the differences in patient selection, the extent of LND, and the presence or absence of distant metastases.

Clinical stage and pathological grade of the tumor is highly predictive of the prevalence of lymph node metastases $(6,12,26)$. When excluding patients with metastatic disease undergoing cytoreductive nephrectomy, the incidence of lymph node metastasis in surgical series decreases to $3-10 \%(1,6,8)$. The relationship of stage and lymph node metastasis has been demonstrated by several authors. Giuliani and colleagues reported a prevalence of nodal involvement of $13.2 \%$ in stages pT $1-2$ and $36.1 \%$ in stage pT3-4 tumors (6). Pantuck and colleagues reported a nodal involvement of $5.2 \%$ versus $23.4 \%$ for T1-2 and T3-4, respectively (12). Similarly, higher tumor grade is also associated with higher rates of positive nodes $(5,6,20)$. Pantuck et al. reported nodal metastasis in $33 \%$ of Fuhrman grade 1-2 tumors (27), as compared with a rate of $68 \%$ in those with grade 3-4 tumors (12).

The incidence of lymph node metastasis at the time of clinical presentation has been steadily decreasing over time as evidenced by longitudinal analysis. When comparing the incidence of positive lymph nodes in Robson et al's early series of radical nephrectomy and the more recent treatment arm of the EORTC 30881 , a drop from $30 \%$ to $3.3 \%$, was observed $(1,14)$.

Another important factor contributing to the lower incidence of positive nodes is the downward stage migration in renal cancer seen with the increased incidental detection of the disease. Studying a series of 309 patients between 1935 and 1965, Skinner et al. found a $7 \%$ prevalence of incidental renal masses (18). More recently, the prevalence of incidental renal tumors reported in a series of 131 patients studied by Jayson and Sanders in 1998 was 61\% (28). Konnak and Grossman also reported on the change in incidental detection of renal tumors over time, from $13 \%$ to $48 \%$, comparing two series of patients treated between 1961 and 1973, and between 1980 and 1984, respectively (29). In addition a simultaneous decline in the stage at the time of diagnosis was also observed, once again confirming the stage migration phenomenon (29). Since one of the most important risk factors for the presence of lymph node metastasis is clinical stage, the increasing detection of incidental small renal tumors is presumably a major etiology for the observed decreasing prevalence of nodal metastasis at the time of presentation.

The importance of the discussion regarding nodal involvement in renal cell carcinoma is based on the fact that the cancer-specific survival is greatly impacted by the presence of lymph node metastasis. Early series reported the 5-year cancer-specific survival rate associated with lymph node metastasis for $\mathrm{RCC}$ ranging from $21 \%$ to $35 \%(14,30)$. More recently, Pantuck et al. and Blute et al. reported in a contemporary series an overall 5-year survival rate of $23 \%$ and $20.9 \%$, respectively $(3,12)$. They also noted on a multivariate analysis that the chance of dying from RCC was 7.87-fold higher with lymph node involvement than without (3), and that patients who did not undergo LND were three times more likely to die than those who did, regardless of the extent of the dissection (12). Despite all controversies about the necessity and extent of the dissection, the presence of lymph node involvement in RCC undoubtedly deserves attention, since the reported poor survival rates can definitely be improved with LND in selected cases. The great challenge is to properly identify those cases that would most benefit from this practice.

\section{Renal Lymphatic Drainage}

Another factor that adds controversy to the indication of performing LND during radical nephrectomy is the unpredictability of renal lymphatic drainage.

The pathways of renal lymphatic drainage were initially described by Parker in 1935, during anatomical studies of the posterior lymphatic channels of the abdomen (31). He noted that the lymphatic drainage of the kidney was neither unique nor specific, and the patterns of drainage could be quite variable. Johnsen and Hellsten (9) in an autopsy study of 554 patients, observed the occurrence of unpredictable patterns of nodal metastasis and the presence of distant metastasis without regional lymph node involvement (9). Saitoh and colleagues in an autopsy study in Japan, analyzing 1828 cases of renal cancer, also observed cases with distant metastasis without regional lymph node invasion (15). They pointed out the im- 
portance of the vascular nature of RCC and hence the predilection for early hematogenic dissemination (32). Clinical series mimic these results $(6,22)$. Vasselli et al. reported an incidence of $53 \%$ of distant metastasis without lymph node invasion (22) and Giuliani and colleagues also observed the extremely poor negative predictive value of regional LND in predicting disease progression (6). It was postulated that the neovascularization of RCC distorts the normal anatomy and renders the lymphatic drainage unpredictable (33). Therefore, defining the role of lymphadenectomy during the surgical treatment of these tumors remains a difficult task and a balance between the morbidity of the procedure and the benefits of its practice must always be sought.

\section{MORBIDITY VERSUS BENEFITS}

\section{Technique and Extent of LND}

The recommended extent of LND has varied from an author to another. The extended dissection, first proposed by Robson et al. in 1969, included "all para-aortic and para-caval lymph nodes, from bifurcation of the aorta to the crus of the diaphragm" (14). Later, new limits were described dependent on laterality. Templates proposed for tumors on the right included the hilar, para-caval, pre-caval, retro-caval, interaortocaval and pre-aortic lymph nodes, whereas for left-sided tumors, inclusion of the hilar, para-aortic, pre-aortic, retro-aortic, interaortocaval and precaval nodes was recommended (34). It is important to note that the primary renal lymphatic drainage on the right is to the interaortocaval lymph nodes, and on the left to the para-aortic nodes.

In practice, many surgeons attempt to decrease morbidity by limiting the extent of dissection. Therefore, a limited regional dissection has been recommended, involving only the para-caval, pre-caval and hilar nodes on the right side, and para-aortic, preaortic and hilar nodes on the left side, particularly in the setting of laparoscopically treated patients where extended node dissection would be technically difficult. Also due to uncertainties about the benefit of LND, disagreement persists about the ideal limits of LND $(3,6,20)$. Given the distribution of lymphatic drainage, the use of limited node dissection in patients with substantial risk of lymph node metastases is likely to result in understaging, particularly on the right side.

Some authors have tried to overcome the divergences in the templates and to improve the staging role of the procedure by analyzing the same issue from a different perspective. Terrone and colleagues reported on the impact of analyzing the number of dissected nodes, instead of anatomical extension of the LND and have found that a minimum number of 13 nodes should be retrieved, in order to properly stage and estimate the prognosis of these patients. This cutoff resulted in a significant increase in the rate of lymph node metastasis found (20). Thus, based on this data, the best approach to effectively yield an adequate lymphadenectomy and optimize staging, would suggest a dissection of a nodal package extending between the regional and the extended pattern, to assure that the proper number of nodes would be retrieved, irrespective of the specific template limits.

\section{Morbidity}

It seems intuitive that an increased extent of lymph node dissection would also increase the morbidity of the procedure. However, when compared to nephrectomy alone, nephrectomy associated with LND, did not show increased morbidity based on retrospective and prospective data $(1,35)$. Additionally, a direct comparison of various dissection patterns was performed by Siminovitch et al. who reported a group of N0M0 patients, who underwent extended, regional or hilar LND templates. They also failed to demonstrate any difference in the morbidity or survival rates among these groups (17). Several other large retrospective series have likewise failed to demonstrate any difference in morbidity rates, as related to the extent of dissection $(2,8,12,16,23,30,36)$.

The most common complications related to the surgical management of RCC are lymphocele, chylous ascites (36), bleeding from lumbar or great vessels, and injury to adjacent organs. However, it is difficult to establish the direct correlation of these events with the LND per se. The EORTC 30881 also addressed this issue and did not show any difference in complications rates, but an increased blood loss was observed among those undergoing LND (1).

Although a significant morbidity is not demonstrated in the literature, LND is still a highly 
complex procedure and because it carries a great potential risk for serious intraoperative life-threatening complications, it should be performed only by skilled, well-trained surgeons, who are familiar with retroperitoneal dissections. In addition to providing surgical expertise, urologists should carefully identify only those candidates in whom there is a clear benefit in performing the LND.

\section{Benefits}

Throughout the years, three potential benefits of LND at the time of nephrectomy have been evaluated: 1) improved staging and prognostication; 2) improved survival following surgery; and most recently 3) improved response to systemic therapy. Given the limitations of the inconsistent lymphatic anatomy described above, the accuracy of staging and the therapeutic value of the procedure in the setting of radiologically normal lymph nodes are highly dependent upon the rigor of dissection utilized and the pathological features of the disease. Recently the practice of LND in localized renal tumors has not showed significant benefit. It is also questionable whether improved staging accuracy is important given the absence of efficacious adjuvant therapies for the disease $(37,38)$. The recent approval in the United States of novel tyrosine kinase inhibitors for the treatment of advanced renal cancer will likely offer an opportunity for the adjuvant treatment of high risk pathology and for a rebirth of LND as staging and/or cytoreductive procedure (39-41).

\section{Imaging Techniques and Staging role of LND}

Although imaging advancements allow detection of nodules as small as $0.5 \mathrm{~cm}$ in the retroperitoneum, there is no imaging method that can confidently differentiate enlarged inflammatory nodes from metastatic ones in RCC $(20,42)$. Studer et al. reported an incidence of only $42 \%$ of histologically positive nodes in his series of patients with preoperatively enlarged nodes seen on computed tomography (CT) scan (19). The sensitivity of CT for enlarged nodal masses greater than $1 \mathrm{cc}$ is higher than $95 \%$ (1), but the low specificity of this finding and the poor predictive value of the method could argue both in favor and against routine LND in these patients. In fact, Studer et al. found that nodes detected by CT, that measured between $1 \mathrm{~cm}$ and $2.2 \mathrm{~cm}$ were more likely to be inflammatory (19). Because conventional imaging is unable to reliably discern lymph node metastasis from non-malignant lymph node enlargement, routine LND is recommended for any individual with radiologically identified lymphadenopathy.

\section{Benefits for Patients with Localized Tumors}

The practice of LND in localized renal tumors has not shown significant benefit. The low incidence of positive nodes, reported to range from $0.4 \%$ in the UCLA data, up to $3.3 \%$ in other series $(1,10,12,43)$, and the lack of survival advantage demonstrated in randomized trial (1) have favored the omission of routine LND in localized tumors with no suspicious nodes in the preoperative imaging. Moreover, the increasing popularity and the successful oncological outcomes of minimally invasive methods and nephron-sparing techniques have also contributed to the decreased enthusiasm for LND in early stages of the disease.

The staging role of LND is also questionable given the absence of effective adjuvant therapies for RCC $(37,38)$. As data regarding adjuvant strategies continues to improve, offering routine LND to high risk patients, defined as those with large tumors (particularly clinical stage $=\mathrm{T} 3$ ), high nuclear grade (Fuhrman's grade) (27), symptoms, and poor performance status $(44,45)$, remains a topic of debate. These individuals have a reported incidence of positive nodes approaching 10\% (46) and thus these patients warrant further attention in future clinical trials.

The application of a risk classification strategy, according to the presence of predictive risk factors, has been proposed as a means of identifying those patients at a higher risk of regional lymph node involvement, that are most likely to benefit from LND. The only study that brought insight into such risk factors was published by Blute et al.(3). Using a multivariate model to identify pathologic features of the primary tumor that were independent predictors of increased risk of having positive nodes in non-metastatic RCC, they identified 5 risk factors: clinical stage (T3-T4), size of the tumor $(>10 \mathrm{~cm}$ ), tumor grade (Fuhrman III-IV) (27), presence of sarcomatoid differentiation and presence of necrosis (3). The presence of two or 
Lymph Node Dissection for RCC in the Modern Era

more of these factors was associated with a 15 -fold higher incidence of regional lymph node involvement. While provocative, the limitation of this approach is the lack of pre-treatment factors for segregating risk. In the absence of good pathologic support, its application could be difficult.

\section{Benefits for Patients with Nodal Metastasis Only}

Little controversy exists about recommending LND in those with isolated positive nodes without distant metastasis. Although this situation is usually found in between $0.9 \%$ and $10 \%$ of the cases, as shown in Table-2 $(1,6,9,10,12,17,25)$, it may reach rates up to $20.4 \%$, as demonstrated by Terrone et al., using extended templates of dissection and retrieving more than 12 nodes (20). Additionally, in spite of the vast majority of patients who have positive nodes also present with concurrent distant metastasis (58-95\% of cases), and exclusive nodal disease is a situation difficult to detect, the survival of this group when treated with LND associated with radical nephrectomy is superior to that of nephrectomy alone (2). Moreover, the survival of this subset of patients who undergo LND associated with radical nephrectomy is far superior to those with distant metastasis, and more closely approximates that of those in the T3N0M0 stage disease $(6,13)$. Data from Giuliani et al. shows that survival rates of this group of patients are $47.9 \%$ and $31.9 \%$, at 5 and 10 years of follow-up, respectively, in comparison to the 7\% 5-year survival rate of patients with distant metastases (6). Peters and Brown also demonstrated an improved survival associating LND with nephrectomy, which increased the survival rates from $56.5 \%$ to $87.5 \%$ at 1 year and from $25.79 \%$ to $43.75 \%$ at 5 years follow-up (13). In this situation, there is little controversy among the experts, and the LND must be performed with curative intent, therefore using an extended template (8). The challenge lies in identifying those cases preoperatively. Perhaps the lack of the proven morbidity associated with the LND, allows for a more liberal indication for the dissection of all suspicious nodes identified before or during surgery. Moreover, the incidental finding of suspicious nodes at radical nephrectomy should also be managed according to this same rationale, since the EORTC trial demonstrated that, despite only $16 \%$ of nodes removed due to suspicious palpation were positive, it was yet significantly more, than the $1 \%$ found to be positive, in those patients who underwent routine dissection (1).

\section{Benefits for Patients with Systemic Metastasis}

With the recent advances in systemic therapies using cytokines and tyrosine kinase inhibitors,

Table 2 - Prevalence of $N+M 0$ disease.

\begin{tabular}{cccccc}
\hline Year & Author (Ref.) & Study Design & $\begin{array}{c}\text { Number of Cases / } \\
\text { Groups Included }\end{array}$ & LND Technique & Prevalence of N+ \\
\hline 1982 & Siminovitch (17) & retrospective & $102 / \mathrm{T} 1-2 \mathrm{~N} 0 \mathrm{M} 0$ & mixed & $8.8 \%$ \\
1990 & Giuliani (6) & retrospective & $200 /$ all stages & extended & $10.0 \%$ \\
1997 & Johnsen \& Hellsten (9) & autopsy & $554 /$ all stages & autopsy & $0.9 \%$ \\
1997 & Giberti (25) & retrospective & $328 /$ all stages & regional / extended & $7.0 \%$ \\
1999 & Blom (1) & prospective & $731 /$ T1-3N0M0 & extended & regional \\
2001 & Minervini (10) & retrospective & $167 /$ TanyN0M0 & mixed & $5.0 \%$ \\
2003 & Pantuck (12) & retrospective & $900 /$ all stages & $5.0 \%$ \\
\hline
\end{tabular}

$L N D=$ lymph node dissection $;+=$ Node positive disease. 
the value of the staging and therapeutic benefit of LND has been increasingly discussed. Although immunotherapy using cytokines such as interleukin-2 and interferon- $\alpha$, alone or in combination, have been widely used as first-line treatment of metastatic RCC, response rates are usually low (5-20\%) with median survival rates ranging 12-17.9 months (or lower in the presence of adverse prognostic factors) and with substantial toxicity (47-51). It has been previously shown that systemic therapy with cytokines improves survival after radical nephrectomy and that lymph node metastases typically have a poor response to chemotherapy and immunotherapy $(12,22,52)$. Vasseli et al. observed that survival rates were longer in patients with systemic disease without retroperitoneal nodal metastasis (median survival of 14.7 months), and that the preoperative presence of retroperitoneal lymphadenopathy predicted a shortened survival. However, when the lymph nodes were completely resected during surgery, overall survival rates of these patients were similar to those without retroperitoneal lymphadenopathy (22). Although there was no benefit in survival rates between groups receiving immunotherapy, this data supports the cytoreductive role of the LND during the management of metastatic RCC with lymph node involvement. A more aggressive surgical approach could positively impact the outcomes of the systemic therapy, reducing the burden of the disease and eliminating the metastatic tissue that is less susceptible to the therapy agents (22). Therefore, the extent of the dissection should always be guided by the rationale of clearing the most as possible the grossly involved nodes and its regional packages. However, as this strategy is still considered palliative, extended (more morbid) templates should be avoided, since these patients benefit from a rapid and uneventful postoperative recovery allowing them to receive systemic therapy as soon as possible.

\section{EVIDENCE-BASED RECOMMENDATIONS}

Based upon the data reviewed above, we propose the following approach for each patient group (Figure-1).

\section{Localized Disease/ Low Risk (T1-2, N0, M0)}

In the contemporary age of decreasing incidence of nodal metastasis, lymph node dissection is of little value in this group. Despite the low morbidity rates, the majority of practitioners agree that there is no indication for LND in these patients, as no survival benefit has been demonstrated for LND with localized RCC.

\section{Localized Disease/ High Risk (T3-4, N0, M0)}

In this subset of tumors lies the greatest controversy. Most urologists generally do not perform LND in this situation because of the substantial risk of concomitant hematogenic metastasis regardless of the state of the lymph nodes, as well as due to the lack of validated criteria to predict nodal metastasis. However, with the poor negative predictive value of the imaging examinations and the promising emerging targeted therapies, the staging role of the LND again becomes a reasonable argument. We recommend regional LND in those patients that present with the risk factors aforementioned, including the hilar and para-aortic nodes for tumors on the left side, and the hilar, para-caval, retro-caval and interaortocaval nodes on the right side, from the crus of the diaphragm until the emergence of the inferior mesenteric artery. In the absence of any risk factors or incidental suspicious lymphadenopathy, probably any LND beyond the hilar nodes level is not justified.

\section{Nodal Metastasis Only (Tany, N+, M0)}

It is essential to first assure accurate preoperative staging and rule out any evidence of distant metastasis. The extended LND is indicated in these patients who, with LND, have survival outcomes similar to the T3N0M0 group. In addition to grossly enlarged nodes, the extended dissection must include all nodes anterior and posterior to the aorta and inferior vena cava, from the crus of the diaphragm until the bifurcation of the great vessels, including the interaortocaval nodes for tumors on the right side. The type of dissection should also be dependent on comorbidities and performance status, in order to select those patients who are more likely to tolerate the extended procedure. The timing of the surgical management in this setting is the key to successfully achieve a cure in these patients. 


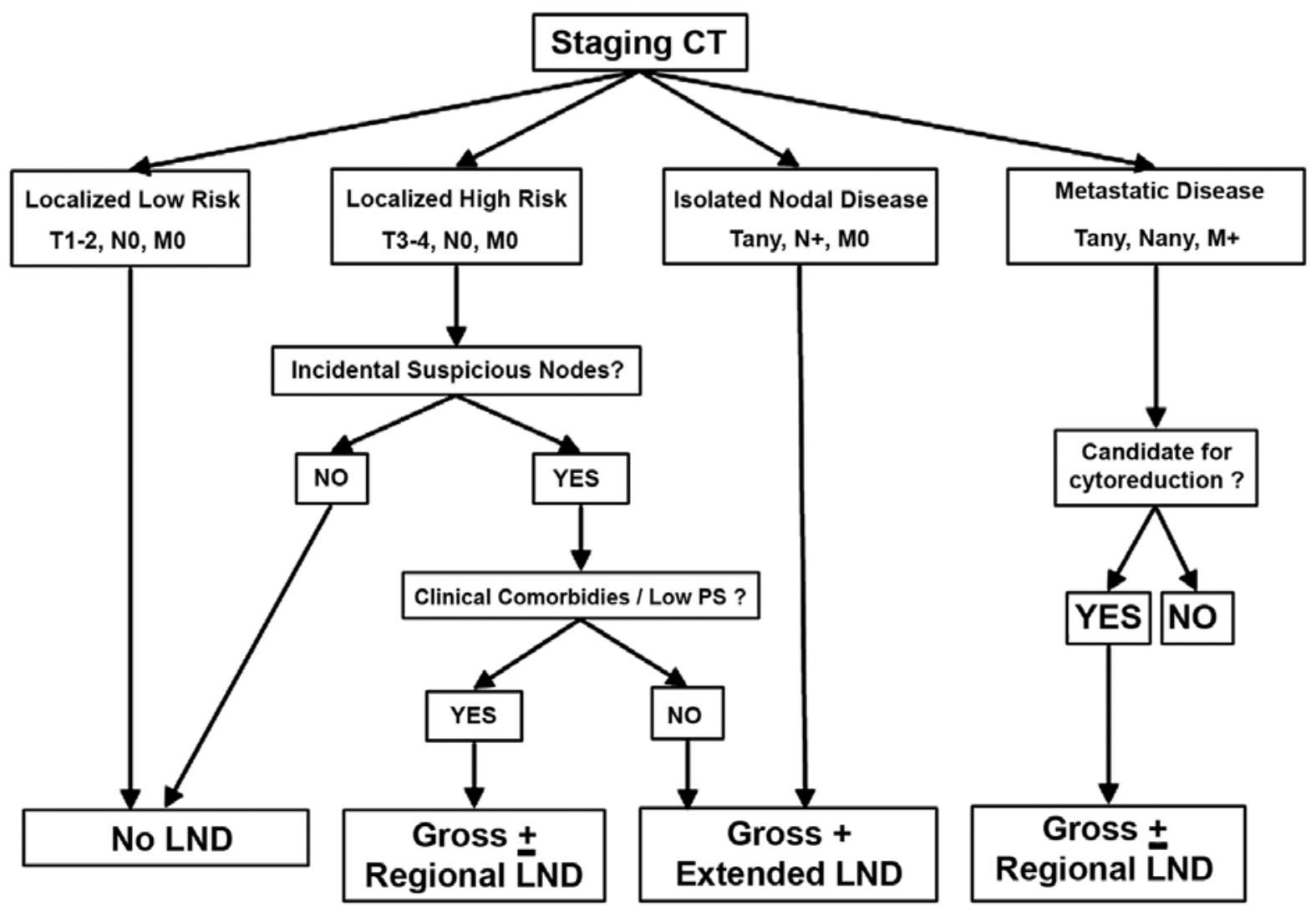

CT: computed tomography; TNM (AJCC 2002); LND: lymph node dissection; PS: Performance status.

Figure 1 - Lymph node dissection during radical nephrectomy algorithm.

\section{Metastatic Disease (Tany, Nany, M+)}

In this group of patients, the cytoreductive approach is beneficial and will likely improve outcomes of systemic therapies. The surgical procedure should include a radical nephrectomy and regional lymphadenectomy including only the grossly positive nodes. As nodal disease shows poor response to immunotherapy, trying to extirpate all visible gross nodal metastasis is always a good practice in selected patients with good performance status, who are likely to tolerate the surgery and recover to receive systemic therapies. Since there is no curative intention, extended patterns of LND are not justified.

\section{CONCLUSIONS}

Lymph node dissection has become an uncommon procedure during the surgical treatment of renal tumors, in the era of small, incidental and early stage renal masses. We have seen an increased rate of surveillance strategies, minimally invasive and nephron-sparing approaches substituting for the classic radical nephrectomy as described by Robson et al. (14). LND in high-risk cases has not shown any proven survival benefit, but in the future may be tailored, based on our better understanding of prognostic factors and the increasing knowledge about 
genetic/molecular events in the carcinogenesis of RCC. To date, there are only two situations in which LND definitely benefits patients: in the presence of nodal involvement without distant metastasis, and as part of a cytoreductive approach. In the next few years, with advancements in novel targeted therapies, further prospective studies will be warranted to redefine the therapeutic/staging roles of LND in the management of renal tumors.

\section{ACKNOWLEDGMENT}

Dr. Guilherme Godoy is recipient of a Grant from Bruce and Cynthia Sherman Fellowship in Urologic Oncology.

\section{CONFLICT OF INTEREST}

None declared.

\section{REFERENCES}

1. Blom JH, van Poppel H, Marechal JM, Jacqmin D, Sylvester R, Schröder FH, et al.: Radical nephrectomy with and without lymph node dissection: preliminary results of the EORTC randomized phase III protocol 30881. EORTC Genitourinary Group. Eur Urol. 1999; 36: 570-5.

2. Freedland SJ, Dekernion JB: Role of lymphadenectomy for patients undergoing radical nephrectomy for renal cell carcinoma. Rev Urol. 2003; 5: 191-5.

3. Blute ML, Leibovich BC, Cheville JC, Lohse CM, Zincke H: A protocol for performing extended lymph node dissection using primary tumor pathological features for patients treated with radical nephrectomy for clear cell renal cell carcinoma. J Urol. 2004; 172: 465-9.

4. Dekernion JB, Ramming KP, Smith RB: The natural history of metastatic renal cell carcinoma: a computer analysis. J Urol. 1978; 120: 148-52.

5. Ditonno P, Traficante A, Battaglia M, Grossi FS, Selvaggi FP: Role of lymphadenectomy in renal cell carcinoma. Prog Clin Biol Res. 1992; 378: 169-74.

6. Giuliani L, Giberti C, Martorana G, Rovida S: Radical extensive surgery for renal cell carcinoma: long-term results and prognostic factors. J Urol. 1990; 143: 46873; discussion 473-4.
7. Golimbu M, Joshi P, Sperber A, Tessler A, Al-Askari S, Morales P: Renal cell carcinoma: survival and prognostic factors. Urology. 1986; 27: 291-301.

8. Herrlinger A, Schrott KM, Schott G, Sigel A: What are the benefits of extended dissection of the regional renal lymph nodes in the therapy of renal cell carcinoma. J Urol. 1991; 146: 1224-7.

9. Johnsen JA, Hellsten S: Lymphatogenous spread of renal cell carcinoma: an autopsy study. J Urol. 1997; 157: 450-3.

10. Minervini A, Lilas L, Morelli G, Traversi C, Battaglia $\mathrm{S}$, Cristofani R, et al.: Regional lymph node dissection in the treatment of renal cell carcinoma: is it useful in patients with no suspected adenopathy before or during surgery? BJU Int. 2001; 88: 169-72.

11. Moudouni SM, Tazi Mokha K, Nouri M, Lrhorfi MH, Koutani A, Iben Attya Andaloussi A, et al.: Renal cancer in adults. Review of 68 cases. Ann Urol (Paris). 1999; 33: 395-401.

12. Pantuck AJ, Zisman A, Dorey F, Chao DH, Han KR, Said J, et al.: Renal cell carcinoma with retroperitoneal lymph nodes: role of lymph node dissection. J Urol. 2003; 169: 2076-83.

13. Peters PC, Brown GL: The role of lymphadenectomy in the management of renal cell carcinoma. Urol Clin North Am. 1980; 7: 705-9.

14. Robson CJ, Churchill BM, Anderson W: The results of radical nephrectomy for renal cell carcinoma. J Urol. 1969; 101: 297-301.

15. Saitoh H, Nakayama M, Nakamura K, Satoh T: Distant metastasis of renal adenocarcinoma in nephrectomized cases. J Urol. 1982; 127: 1092-5.

16. Schafhauser W, Ebert A, Brod J, Petsch S, Schrott KM: Lymph node involvement in renal cell carcinoma and survival chance by systematic lymphadenectomy. Anticancer Res. 1999; 19: 1573-8.

17. Siminovitch JP, Montie JE, Straffon RA: Lymphadenectomy in renal adenocarcinoma. J Urol. 1982; 127: 1090-1.

18. Skinner DG, Colvin RB, Vermillion CD, Pfister RC, Leadbetter WF: Diagnosis and management of renal cell carcinoma. A clinical and pathologic study of 309 cases. Cancer. 1971; 28: 1165-77.

19. Studer UE, Scherz S, Scheidegger J, Kraft R, Sonntag $\mathrm{R}$, Ackermann D, et al.: Enlargement of regional lymph nodes in renal cell carcinoma is often not due to metastases. J Urol. 1990; 144: 243-5.

20. Terrone C, Guercio S, De Luca S, Poggio M, Castelli E, Scoffone C, et al.: The number of lymph nodes examined and staging accuracy in renal cell carcinoma. BJU Int. 2003; 91: 37-40. 
21. Tsukamoto T, Kumamoto Y, Miyao N, Yamazaki K, Takahashi A, Satoh M: Regional lymph node metastasis in renal cell carcinoma: incidence, distribution and its relation to other pathological findings. Eur Urol. 1990; 18: 88-93.

22. Vasselli JR, Yang JC, Linehan WM, White DE, Rosenberg SA, Walther MM: Lack of retroperitoneal lymphadenopathy predicts survival of patients with metastatic renal cell carcinoma. J Urol. 2001; 166: 68-72.

23. Waters WB, Richie JP: Aggressive surgical approach to renal cell carcinoma: review of 130 cases. J Urol. 1979; 122: 306-9.

24. Sigel A, Chlepas S, Schrott KM, Hermanek P: Surgery of the kidney tumor. Chirurg. 1981; 52: 545-53.

25. Giberti C, Oneto F, Martorana G, Rovida S, Carmignani G: Radical nephrectomy for renal cell carcinoma: long-term results and prognostic factors on a series of 328 cases. Eur Urol. 1997; 31: 40-8.

26. Phillips CK, Taneja SS: The role of lymphadenectomy in the surgical management of renal cell carcinoma. Urol Oncol. 2004; 22: 214-23; discussion 223-4.

27. Fuhrman SA, Lasky LC, Limas C: Prognostic significance of morphologic parameters in renal cell carcinoma. Am J Surg Pathol. 1982; 6: 655-63.

28. Jayson M, Sanders H: Increased incidence of serendipitously discovered renal cell carcinoma. Urology. 1998; 51: 203-5.

29. Konnak JW, Grossman HB: Renal cell carcinoma as an incidental finding. J Urol. 1985; 134: 1094-6.

30. Skinner DG, Vermillion CD, Colvin RB: The surgical management of renal cell carcinoma. J Urol. 1972; 107: 705-10.

31. Parker AE: Studies on the main posterior lymph channels of the abdomen and their connections with the lymphatics of the genitourinary system. Am J Anat. 1935; 56: 409.

32. Saitoh H: Distant metastasis of renal adenocarcinoma in patients with a tumor thrombus in the renal vein and/or vena cava. J Urol. 1982; 127: 652-3.

33. DeKernion JB: Lymphadenectomy for renal cell carcinoma. Therapeutic implications. Urol Clin North Am. 1980; 7: 697-703.

34. Wood DP Jr: Role of lymphadenectomy in renal cell carcinoma. Urol Clin North Am. 1991; 18: 421-6.

35. Carmignani G, Belgrano E, Puppo P, Gilberti C, Cicherro A: Lymphadenectomy in renal cancer. In: Cancer of the Prostate and Kidney (ed.), Pavone-Macaluso M. Smith PH. New York, Plenum Press. 1983, pp. 645-50.
36. Ferrigni RG, Novicki DE: Chylous ascites complicating genitourinary oncological surgery. J Urol. 1985; 134: 774-6.

37. Naito S, Kumazawa J, Omoto T, Iguchi A, Sagiyama K, Osada Y, et al.: Postoperative UFT adjuvant and the risk factors for recurrence in renal cell carcinoma: a long-term follow-up study. Kyushu University Urological Oncology Group. Int J Urol. 1997; 4: 8-12.

38. Pizzocaro G, Piva L, Colavita M, Ferri S, Artusi $\mathrm{R}$, Boracchi $\mathrm{P}$, et al.: Interferon adjuvant to radical nephrectomy in Robson stages II and III renal cell carcinoma: a multicentric randomized study. J Clin Oncol. 2001; 19: 425-31.

39. Escudier B, Eisen T, Stadler WM, Szczylik C, Oudard $\mathrm{S}$, Siebels M, et al.: Sorafenib in advanced clear-cell renal-cell carcinoma. N Engl J Med. 2007; 356: 12534. Erratum in: N Engl J Med. 2007; 357: 203.

40. Hudes G, Carducci M, Tomczak P, Dutcher J, Figlin R, Kapoor A, et al.: Temsirolimus, interferon alfa, or both for advanced renal-cell carcinoma. N Engl J Med. 2007; 356: 2271-81.

41. Motzer RJ, Hutson TE, Tomczak P, Michaelson MD, Bukowski RM, Rixe O, et al.: Sunitinib versus interferon alfa in metastatic renal-cell carcinoma. N Engl J Med. 2007; 356: 115-24.

42. Bechtold RE, Zagoria RJ: Imaging approach to staging of renal cell carcinoma. Urol Clin North Am. 1997; 24: 507-22.

43. Mickisch GH: Lymphatic metastases in renal cell carcinoma. What is the value of operation and adjuvant therapy? Urologe A. 1999; 38: 326-31.

44. Zisman A, Pantuck AJ, Dorey F, Chao DH, Gitlitz BJ, Moldawer N, et al.: Mathematical model to predict individual survival for patients with renal cell carcinoma. J Clin Oncol. 2002; 20: 1368-74.

45. Zisman A, Pantuck AJ, Wieder J, Chao DH, Dorey F, Said JW, et al.: Risk group assessment and clinical outcome algorithm to predict the natural history of patients with surgically resected renal cell carcinoma. J Clin Oncol. 2002; 20: 4559-66.

46. Zisman A, Pantuck AJ, Belldegrun AS: Lymph Node Dissection in Renal and Adrenal Tumors: Biology and Management. New York, Oxford University Press. 2003; p. 318.

47. Fisher RI, Rosenberg SA, Fyfe G: Long-term survival update for high-dose recombinant interleukin-2 in patients with renal cell carcinoma. Cancer J Sci Am. 2000; 6 (Suppl 1): S55-7.

48. McDermott DF, Regan MM, Clark JI, Flaherty LE, Weiss GR, Logan TF, et al.: Randomized phase III 
trial of high-dose interleukin-2 versus subcutaneous interleukin-2 and interferon in patients with metastatic renal cell carcinoma. J Clin Oncol. 2005; 23: 133-41. Erratum in: J Clin Oncol. 2005; 23: 2877.

49. Motzer RJ, Murphy BA, Bacik J, Schwartz LH, Nanus DM, Mariani T, et al.: Phase III trial of interferon alfa2 a with or without 13 -cis-retinoic acid for patients with advanced renal cell carcinoma. J Clin Oncol. 2000; 18: 2972-80.

50. Négrier S, Escudier B, Gomez F, Douillard JY, Ravaud A, Chevreau C, et al.: Prognostic factors of survival and rapid progression in 782 patients with metastatic renal carcinomas treated by cytokines: a report from the Groupe Français d'Immunothérapie. Ann Oncol. 2002; 13: 1460-8.

51. Negrier S, Escudier B, Lasset C, Douillard JY, Savary $\mathrm{J}$, Chevreau C, et al.: Recombinant human interleukin-2, recombinant human interferon alfa-2a, or both in metastatic renal-cell carcinoma. Groupe Français d'Immunothérapie. N Engl J Med. 1998; 338: 12728.

52. Flanigan RC, Salmon SE, Blumenstein BA, Bearman SI, Roy V, McGrath PC, et al.: Nephrectomy followed by interferon alfa- $2 \mathrm{~b}$ compared with interferon alfa- $2 \mathrm{~b}$ alone for metastatic renal-cell cancer. N Engl J Med. 2001; 345: 1655-9. $\overline{\text { Accepted: }}$

April 4, 2008

Correspondence address:

Dr. Samir S. Taneja

Department of Urology

New York School of Medicine

150 East 32nd street, 2nd floor

New York, NY, 10016, USA

Fax: + 1646 825-6394

E-mail: samir.taneja@nyumc.org 